\title{
Employing thin HPGe detectors for gamma-ray imaging
}

K. Vetter, L. Milhailescu, K. Ziock, M. Burks, E. Hull, N. Madden, R. Pehl

This article was submitted to Workshop on Unattended Radiation Sensor Systems for Remote Applications, Washington, D. C., April 15-17, 2002

\section{May 2, 2002}

Lawrence

Livermore

National

Laboratory

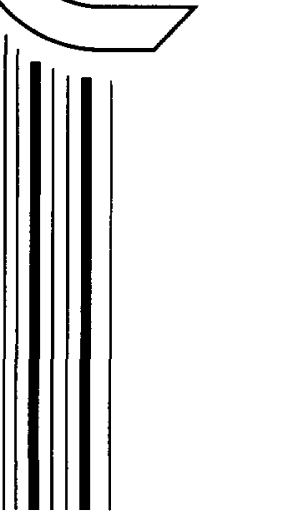




\section{DISCLAIMER}

This document was prepared as an account of work sponsored by an agency of the United States Government. Neither the United States Government nor the University of California nor any of their employees, makes any warranty, express or implied, or assumes any legal liability or responsibility for the accuracy, completeness, or usefulness of any information, apparatus, product, or process disclosed, or represents that its use would not infringe privately owned rights. Reference herein to any specific commercial product, process, or service by trade name, trademark, manufacturer, or otherwise, does not necessarily constitute or imply its endorsement, recommendation, or favoring by the United States Government or the University of California. The views and opinions of authors expressed herein do not necessarily state or reflect those of the United States Government or the University of California, and shall not be used for advertising or product endorsement purposes.

This is a preprint of a paper intended for publication in a journal or proceedings. Since changes may be made before publication, this preprint is made available with the understanding that it will not be cited or reproduced without the permission of the author.

This work was performed under the auspices of the United States Department of Energy by the University of California, Lawrence Livermore National Laboratory under contract No. W-7405-Eng-48.

This report has been reproduced directly from the best available copy.

Available electronically at http://www.doc.gov/bridge

Available for a processing fee to U.S. Department of Energy

And its contractors in paper from

U.S. Department of Energy

Office of Scientific and Technical Information

P.O. Box 62

Oak Ridge, TN 37831-0062

Telephone: (865) 576-8401

Facsimile: (865) 576-5728

E-mail: reports@adonis.osti.gov

Available for the sale to the public from

U.S. Department of Commerce

National Technical Information Service

5285 Port Royal Road

Springfield, VA 22161

Telephone: (800) 553-6847

Facsimile: (703) 605-6900

E-mail: orders@ntis.fedworld.gov

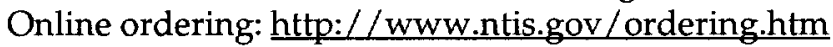

$$
\text { OR }
$$

Lawrence Livermore National Laboratory

Technical Information Department's Digital Library

http://www.llnl.gov/tid/Library.html 


\title{
Employing thin HPGe detectors for gamma-ray imaging
}

\author{
K.Vetter ${ }^{\mathrm{a}}$, L. Mihailescu ${ }^{\mathrm{a}}, \mathrm{K}$. Ziock $^{\mathrm{a}}$, \\ M. Burks ${ }^{b}$, E. Hull ${ }^{\text {, }}$ N. Madden ${ }^{b}$, R. Pehl ${ }^{b}$

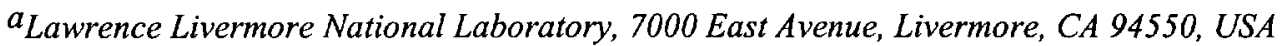

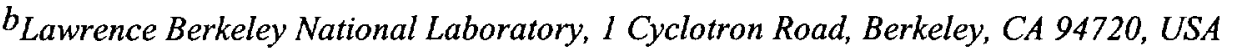

\begin{abstract}
.
We have evaluated a collimator-less gamma-ray imaging system, which is based on thin layers of double-sided strip HPGe detectors. The positions of individual gamma-ray interactions will be deduced by the strip addresses and the Ge layers which fired. Therefore, high bandwidth pulse processing is not required as in thick Ge detectors. While the drawback of such a device is the increased number of electronics channels to be read out and processed, there are several advantages, which are particularly important for remote applications: the operational voltage can be greatly reduced to fully deplete the detector and no high bandwidth signal processing electronics is required to determine positions. Only a charge sensitive preamplifier, a slow pulse shaping amplifier, and a fast discriminator are required on a per channel basis in order to determine photon energy and interaction position in three dimensions. Therefore, the power consumption and circuit board real estate can be minimized. More importantly, since the high bandwidth signal shapes are not used to determine the depth position, lower energy signals can be processed. The processing of these lower energy signals increases the efficiency for the recovery of small angle scattering. Currently, we are studying systems consisting of up to ten $2 \mathrm{~mm}$ thick Ge layers with $2 \mathrm{~mm}$ pitch size. The required electronics of the few hundred channels can be integrated to reduce space and power. We envision applications in nuclear nonproliferation and gamma-ray astronomy where ease of operation and low power consumption, and reliability, are crucial.
\end{abstract}




\section{INTRODUCTION}

The ability to image and characterize known as well as unknown gamma-ray sources finds a variety of applications in national security, such as nuclear nonproliferation and stockpile stewardship programs, in nuclear waste control and monitoring, in nuclear medicine for cancer diagnosis and therapy as well as in astrophysics. Besides these applications, which are based on the natural gamma-ray emission of radioactive material of interest, gamma-ray imaging can be employed in alternate modes, such as particle induced gamma-ray emission, scattering or transmission modes.

Recent advances in the manufacture of two-dimensional segmented semi-conductor detectors enable to determine energies and three-dimensional positions of individual gamma-ray interactions in the detector. Using tracking algorithms, which are based in the underlying interaction processes such as the Compton scattering and the photoelectrical effect, it is possible to determine the time sequence of the interactions. Knowing the energies and positions of the first interactions allows to determine the incident angle of the incoming gamma ray without the use of a collimator which will significantly increase the efficiency a gamma-ray imaging system. However, to realize the full potential of gamma-ray tracking and Compton-tracking based gamma-ray imaging, particularly for low gamma-ray energies, the excellent position and energy resolution of a semi-conductor detector is needed.

In this study we are focusing on the use of thin high-purity Ge (HPGe) detectors in planar geometry, which are equipped with orthogonal strips on each side (so called double-sided strip detector (DSSD)). However, in the following we will briefly explore characteristics of the two possible geometries of HPGe detectors: the planar and the coaxial configuration.

\section{Coaxial vs. planar HPGe detector}

Generally, two-dimensionally segmented HPGe detectors can be built in two different geometries: planar and coaxial.

Coaxial detectors can be built up to diameters of $8 \mathrm{~cm}$ and lengths of $14 \mathrm{~cm}$. The Boron contact at the outside can be segmented longitudinally and azimuthally as shown on the left side of figure 1. The advantage of coaxial detectors is their compactness and therefore their high efficiency in detecting gamma radiation and the large field-of-view (FOV).

Planar detectors can be built up to $20 \mathrm{~mm}$ thick and with an area of up to $90 \times 90 \mathrm{~mm}^{2}$. They can be segmented two-dimensionally either in pixels on one side or in orthogonal strips on each side. Recent advances in detector processing enable to use reliable contacts on both sides of the crystal. To increase efficiency in particular for higher gamma-ray energies several of these planar DSSD detectors can be arranged in a stack.as shown in figure 1 . 
However, for imaging applications the high efficiency of large volume detectors is not the primary goal but the sensitivity to measure a weak signal out of background radiation. Only the capability to provide a spatial resolution as with an imaging device can effectively increase the signal-to-background ratio by distributing the background throughout the solid angle while maintaining the gamma-ray flux of interest locally in one point. Only increasing the efficiency will increase both, the signal and the background.

Ultimately, only an imaging capability is able to provide geometric dimensions and extensions of materials of interest.

To obtain energy and three-dimensional position information of individual interactions as a prerequisite for gamma-ray tracking in a two-dimensionally segmented coaxial detector, in coaxial HPGe detectors the pulse shapes of charge collecting as well as adjacent segments have to be analyzed which requires complex processing of the signals. However, in this way not only the radius can be determined but also the complementary longitudinal and azimuthal positions can be determined to much higher accuracy than the segment size.

The planar geometry is much simpler and allows to obtain positions and energies with higher accuracy than the segmentation size and the crystal thickness with much reduced signal processing. It is even possible to build a stack array of thin planar DSSD detectors, which don't require any complex processing to obtain energy and position of individual interactions. If the detector is thin enough and the pitch size of the strips is sufficiently small to minimize multiple scattering processes in one of these "voxels" and to provide sufficient position resolution just by the dimensions of these "voxels", then just the strip and crystal IDs and their measured energies can be used for tracking.

\section{Features and advantages of thin DSSD HPGe detectors}

In thin planar DSSD HPGe detectors no complex and high-bandwidth related pulse processing is required since only the energy has to be determined. The thickness of the planar crystals and the strip sizes on each side define a three-dimensional "voxel" which is dimensioned to provide sufficient position resolution and to reduce the number of multiple interactions in one "voxel". As indicated above, if an energy is measured in one "voxel" then the position of the interaction is known to the accuracy of this voxel just by it's ID number.

We envision crystal thicknesses of $2 \mathrm{~mm}$ and pitch sizes of $2 \mathrm{~mm}$ and therefore "voxel" sizes of $2 \mathrm{~mm}^{3}$

Assuming a low count-rate environment in which neither timing nor position determination requires a bandwidth in the order of $10 \mathrm{MHz}$ the bandwidth can be lowered to about $100 \mathrm{kHz}$ to provide the necessary shaping to optimize the energy resolution for each "voxel". The costs for the processing electronics can be reduced due the reduced number of parts required and the lower costs of the parts. In addition, the electronics can be built more compact, which is very important for eventually integrating the hundreds of channels. 
Since thin HPGe detectors can be depleted with a few tenth instead of a few hundreds or even thousands volts the high voltage support can be simplified. In particular, the dimensions of the high-voltage filter can be reduced as well as the $Z$ of the material can be lowered to reduce scattering sources for gamma rays outside the detector.

Since no high-power demanding high-bandwidth analog or digital electronics is required the electronics power consumption can be minimized.

FET's and all parts of the electronics can be moved outside the cryostat and can be thermally decoupled from it to minimize necessary cooling of the Ge detector.

By using thin HPGe detectors the requirements for material quality, for example in terms of the concentration and distribution of impurities, can be reduced. Otherwise disturbing effects caused by charge trapping or by material and field inhomogeneities are minimized.

Lowering the requirements for the crystal quality lowers the costs in manufacturing these detectors since Ge material can be used which otherwise would have to be reprocessed to achieve sufficient quality for other detectors.

Since the requirements to obtain a three-dimensional position of interactions is reduced to filtering a signal out of the noise background which can be done very effectively with slow shaping amplifiers much smaller signals can be accepted for the position determination. Since small signals are associated with small energies which themselves are related with small scattering angles, smaller scattering angles can be accepted.

Finally, since the distances between the HPGe wafers can be changed, systems can be optimized for efficiency (small distances) or spatial resolution (large distances).

Figure 1 shows three possible arrangements of two-dimensionally segmented HPGe detectors and a table to compare basic properties of these systems.

\section{Drawbacks of thin DSSD HPGe detectors}

One drawback in using thin DSSD HPGe detectors to obtain energies and positions of individual gamma-ray interactions is the number of required electronics channels. Instead of using in the order of 40 as in a coaxial system or 100 in a mixed planar DSSD system, here we need to equip potentially more than 1000 channels with shaping amplifiers and digitizers. However, since the requirements for processing are low, integrated electronics including amplifiers and digitizers can be built and employed to minimize space requirements. In addition amplifier and digitizer systems can be envisioned in which each channel only requires $20-30 \mathrm{~mW}$ per channel resulting in about $30 \mathrm{~W}$ for a system of 100 channels.

Using thin detectors increases the probability that electrons, which are scattered out of the atom from the interacting gamma ray are escaping the Ge detector or at least the sensitive part of the detector without leaving the full energy. These events reduce the 
efficiency in detecting the full energy of the incident gamma ray and cause an increased background in the gamma-ray spectrum.

Figure 2 shows calculated losses in efficiencies for different thicknesses and numbers of planar HPGe detectors for three different gamma-ray energies: $186 \mathrm{keV}$

$\left({ }^{235} \mathrm{U}\right), 375 \mathrm{keV}\left({ }^{239} \mathrm{Pu}\right)$ and $646 \mathrm{keV}\left({ }^{239} \mathrm{Pu}\right)$. For example, in our proposed design of 10 layers of $2 \mathrm{~mm}$ thick HPGe detectors we expect to loose about $15 \%$ of full-energy events at a gamma-ray energy of $646 \mathrm{keV}$.

The advantage of the excellent energy resolution of $\mathrm{Ge}$ is associated with the requirement to cool the Ge crystal close to liquid nitrogen temperatures to reduce the thermal noise. However, recent developments in cooling technologies allow now to cool Ge detectors by electromechanical means and avoiding the many problems of liquid nitrogen, particularly for remote detection applications. The cryostat, wiring and feedthroughs have to designed to minimize the thermal power consumption. We envision that a system can be designed and built with a heat load, which requires less than $30 \mathrm{~W}$ electrical power.

\section{CONCLUSIONS}

We have studied an array of thin DSSD HPGe detectors to be used as gamma-ray imager. Three-dimensional positions of gamma-ray interactions are measured by the strip and crystal ID. Therefore, no complex signal processing is required to obtain positions. Only low bandwidth analog electronics is necessary to obtain energy for each "voxel" fired. Positions and energies from such an array can be used to track the interactions of the gamma ray through the detector and to determine the incident angle of the incoming gamma ray which enables collimator-less gamma-ray imaging.

Low bandwidth electronics as well as thin planar HPGe detectors can be manufactured with very low cost and can be operated very reliably due to the reduced sensitivity to detector and electronics quality. Since only a signal which is above the noise is required to determine the position of the scattering process, events with smaller scattering angles can be accepted.

In summary, the proposed array of thin two-dimensionally segmented HPGe detectors represents an appealing approach for remote gamma-ray imaging applications which realizes the full potential of gamma-ray tracking

by minimizing costs, power and signal processing complexity. 
Figure 1: Comparison of different HPGe detector arrangements

Closed-ended coaxial HPGe detector
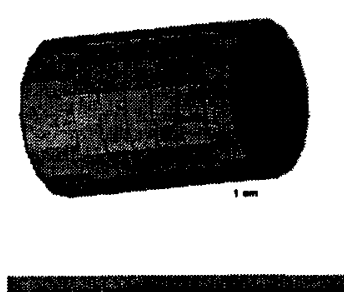

Two-crystal stack of "thick" planar DSSD HPGe detectors
Many-crystal stacks of "thin" planar DSSD HPGe detectors

\begin{tabular}{|c|c|c|c|}
\hline $\begin{array}{c}\text { Signal processing } \\
\text { complexity }\end{array}$ & $\begin{array}{c}\text { Very complex, has to be done } \\
\text { in digital electronics }\end{array}$ & $\begin{array}{c}\text { Medium, depth and potentially } \\
\mathrm{x} \text { and } \mathrm{y} \text { can be done in analog } \\
\text { electronics }\end{array}$ & $\begin{array}{c}\text { Very low, only analog } \\
\text { electronics required for energy } \\
\text { determination }\end{array}$ \\
\hline $\begin{array}{c}\text { Bandwidth of processing } \\
\text { electronics }\end{array}$ & High & High & Low \\
\hline $\begin{array}{c}\text { Number of electronics } \\
\text { channels }\end{array}$ & Low & Medium & High \\
\hline Power & High & Low & Low \\
\hline Costs & High & High & \\
\hline
\end{tabular}

Figure 2:

Calculated efficiencies taking into account electrons which leave the sensitive volume of the HPGe detector, indicating the drop in efficiency due to this effect. Shown are 5 different stacked planar DSSD HPGe systems with wafer thicknesses varying in between $1 \mathrm{~mm}$ and $10 \mathrm{~mm}$ and numbers of layers in between 20 and 2, respectively. Efficiencies are plotted for three gammaray energies: $186 \mathrm{keV}, 375 \mathrm{keV}$ and $646 \mathrm{keV}$. While in the two-layer system the maximum loss is about $4 \%$ at $646 \mathrm{keV}$, in a $\mathbf{2 0}$ layer system about $28 \%$ of the fullenergy efficiency will be lost.

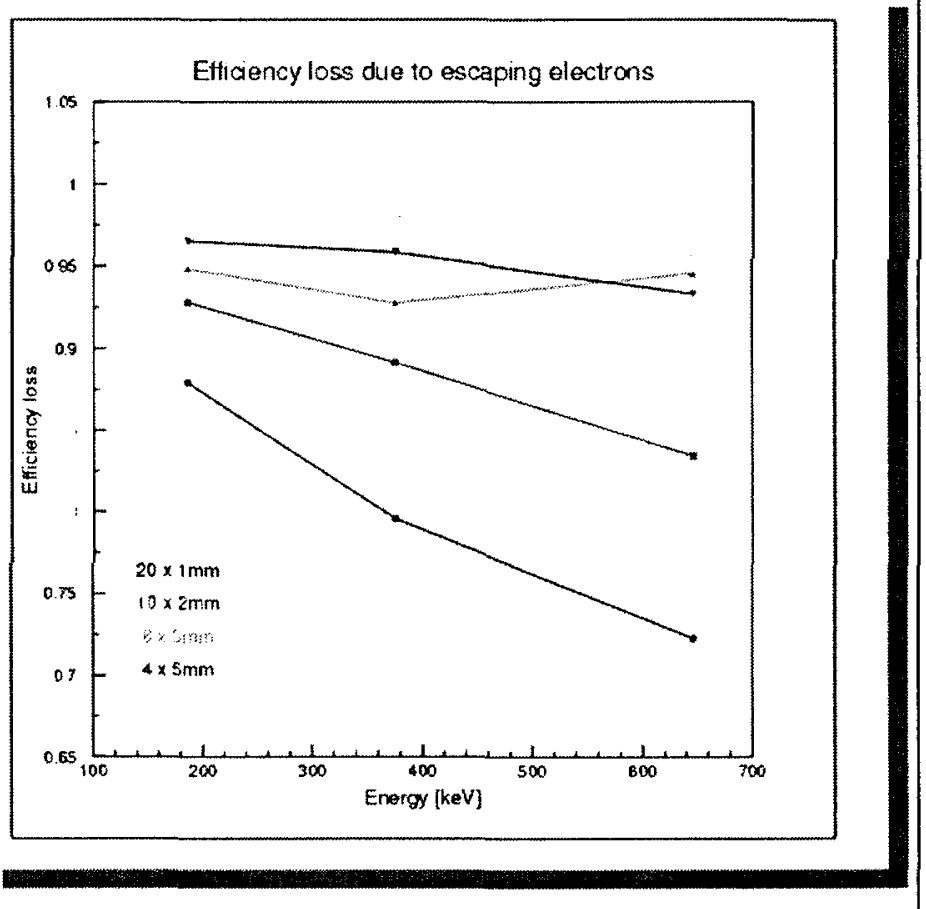




\section{ACKNOWLEDGMENTS}

The work was performed under the auspices of the U.S. Department of Energy by University of California Lawrence Livermore National Laboratory under contract No. W-7405-Eng-48. 